\title{
Differential diagnostic value of total alpha- synuclein assay in the cerebrospinal fluid between Alzheimer's disease and dementia with Lewy bodies from the prodromal stage
}

Olivier Bousiges ${ }^{1,2,3,4^{*}}$ (D), Nathalie Philippi ${ }^{4,5}$, Thomas Lavaux ${ }^{1}$, Armand Perret-Liaudet ${ }^{6,7,8}$, Ingolf Lachmann ${ }^{9}$, Caroline Schaeffer-Agalède ${ }^{1}$, Pierre Anthony ${ }^{10}$, Anne Botzung ${ }^{4}$, Lucie Rauch ${ }^{4}$, Barbara Jung ${ }^{4}$,

Paulo Loureiro de Sousa ${ }^{5}$, Catherine Demuynck ${ }^{4}$, Catherine Martin-Hunyadi ${ }^{4}$, Benjamin Cretin ${ }^{4,5}$ and Frédéric Blanc ${ }^{4,5}$

\begin{abstract}
Background: Several studies have investigated the value of alpha-synuclein assay in the cerebrospinal fluid (CSF) of Alzheimer's disease (AD) and dementia with Lewy bodies (DLB) patients in the differential diagnosis of these two pathologies. However, very few studies have focused on this assay in AD and DLB patients at the MCl stage.

Methods: All patients were enrolled under a hospital clinical research protocol from the tertiary Memory Clinic (CM2R) of Alsace, France, by an experienced team of clinicians. A total of 166 patients were included in this study: 21 control subjects (CS), 51 patients with DLB at the prodromal stage (pro-DLB), 16 patients with DLB at the demented stage (DLB-d), 33 AD patients at the prodromal stage (pro-AD), 32 AD patients at the demented stage $(A D-d)$, and 13 patients with mixed pathology (AD+DLB). CSF levels of total alpha-synuclein were assessed using a commercial enzyme-linked immunosorbent assay (ELISA) for alpha-synuclein (AJ Roboscreen). Alzheimer's biomarkers (t-Tau, P-Tau, A 342 , and A 340 ) were also measured.

Results: The alpha-synuclein assays showed a significant difference between the AD and DLB groups. Total alphasynuclein levels were significantly higher in AD patients than in DLB patients. However, the ROC curves show a moderate discriminating power between $A D$ and DLB $(A \cup C=0.78)$ which does not improve the discriminating power of the combination of Alzheimer biomarkers (AUC $=0.95$ with or without alpha-synuclein). Interestingly, the levels appeared to be altered from the prodromal stage in both $A D$ and DLB.
\end{abstract}

(Continued on next page)

\footnotetext{
* Correspondence: olivier.bousiges@chru-strasbourg.fr

'Laboratory of Biochemistry and Molecular Biology, University Hospital of Strasbourg, 67000 Strasbourg, France

²aboratoire de Neurosciences Cognitives et Adaptatives (LNCA), University of Strasbourg, 67000 Strasbourg, France

Full list of author information is available at the end of the article
}

C C The Author(s). 2020 Open Access This article is licensed under a Creative Commons Attribution 4.0 International License, which permits use, sharing, adaptation, distribution and reproduction in any medium or format, as long as you give appropriate credit to the original author(s) and the source, provide a link to the Creative Commons licence, and indicate if changes were made. The images or other third party material in this article are included in the article's Creative Commons licence, unless indicated otherwise in a credit line to the material. If material is not included in the article's Creative Commons licence and your intended use is not permitted by statutory regulation or exceeds the permitted use, you will need to obtain permission directly from the copyright holder. To view a copy of this licence, visit http://creativecommons.org/licenses/by/4.0/. The Creative Commons Public Domain Dedication waiver (http://creativecommons.org/publicdomain/zero/1.0/) applies to the data made available in this article, unless otherwise stated in a credit line to the data. 
(Continued from previous page)

Conclusions: The modification of total alpha-synuclein levels in the CSF of patients occurs early, from the prodromal stage. The adding of alpha-synuclein total to the combination of Alzheimer's biomarker does not improve the differential diagnosis between $A D$ and DLB.

Trial registration: ClinicalTrials.gov, NCT01876459 (AlphaLewyMa)

Keywords: Dementia with Lewy bodies, Alzheimer's disease, Prodromal, Dementia, Cerebrospinal fluid biomarkers, Total alpha-synuclein

\section{Background}

Dementia with Lewy bodies (DLB) is the most frequent dementia after Alzheimer's disease (AD). The clinical diagnosis of DLB is well defined and regularly revised [1-4]. Despite the prevalence of DLB, only one third of patients are correctly diagnosed, leaving two thirds of these patients undiagnosed or misdiagnosed [5]. DLB is complicated to diagnose due to its similarity to $\mathrm{AD}$ and Parkinson's disease (PD). DLB is close to AD because of cognitive decline (episodic memory, working memory, executive functions) and to PD because of parkinsonism and for the pathophysiological aspect because of the alpha-synuclein ( $\alpha$-syn) aggregation. What happens first in DLB is the cognitive decline, which explains the frequent misdiagnosis with $\mathrm{AD}$. Furthermore, the cognitive and motor symptoms found in DLB can be found in other diseases, which makes differential diagnosis complex. Like other neurodegenerative diseases, DLB progresses insidiously and slowly to a demented state. We now know the importance of early treatment in neurodegenerative disease. Consequently, when effective treatment arrives on the market, we will need to be able to treat patients at a prodromal stage. It is therefore important to be able to diagnose these patients early.

The prodromal stage of DLB (pro-DLB), also called mild cognitive impairment due to Lewy bodies (MCILB), has recently been described in detail: the first criteria of this prodromal stage are similar to the stage of dementia with the difference that decrease in functional capacity is either non-existent or minimal [6].

It is challenging to diagnose DLB at an early stage and, if we add to this the neurological comorbidities that are common in the elderly and more particularly with DLB [7], it is easy to understand the difficulty in diagnosing this type of disease. For all these reasons, it is clear that specific biomarkers need to be found to allow the differential diagnosis of DLB.

To date, many studies have focused on biomarkers used in clinical routine, i.e., Alzheimer's biomarkers ( $t$ Tau, P-Tau, A $\beta 42, A \beta 40$; for a review, see [8]). These studies have shown the great interest of these biomarkers, especially $t-T a u, P-T a u$, and the ratio $A \beta 42$ / $\mathrm{A} \beta 40$, in the differential diagnosis between $\mathrm{AD}$ and $\mathrm{DLB}$, especially at the prodromal stage, where the differential diagnosis is even more delicate $[9,10]$.

DLB and PD, as well as multiple system atrophy (MSA), have one thing in common, namely the $\alpha$-syn aggregation leading to Lewy body formation. That is why these pathologies are part of a group of disorders known as synucleinopathies. Based on these aggregative phenomena and on the way in which amyloid and Tau biomarkers are used in $\mathrm{AD}$, these $\alpha$-syn-related proteins could be of interest in the differential diagnosis of DLB. Studies that have included the measurement of total $\alpha$ syn are relatively numerous and not always consensual.

The aim of our study was therefore to determine the discriminating ability of the $\alpha$-syn assay in cerebrospinal fluid (CSF), without or in combination with the standard AD-related biomarkers, between DLB and AD patients, in both demented and mild cognitive impairment (MCI) patients.

\section{Methods \\ Patients}

All patients were enrolled under a hospital clinical research protocol called AlphaLewyMA (registered in ClinicalTrials. gov: https://clinicaltrials.gov/ct2/show/NCT01876459) from the tertiary Memory Clinic (CM2R) of Alsace by an experienced team of neurologists, geriatricians, and neuropsychologists between June 2013 and June 2018. The CM2R of Alsace comprises 3 different centers, two at the University Hospitals of Strasbourg (CHU Hautepierre and Hôpital de la Robertsau) and one at Hôpitaux Civils de Colmar. Patients underwent detailed clinical evaluation, a large neuropsychological evaluation, blood examination, brain MRI (3 Tesla), and lumbar puncture for CSF biomarkers as previously described [11].

DLB patients were selected according to McKeith's criteria (probable DLB, based on the existence of two core symptoms in addition to cognitive decline) for DLB demented (DLB-d) and prodromal DLB (pro-DLB) patients also called mild cognitive impairment with Lewy bodies (MCI-LB) [3, 6]. To note, Parkinsonism is present in $81.6 \%$ of the pro-DLB patients. However, Parkinsonism is in any case very subtle. For information, fluctuations were assessed with the Mayo Clinic Fluctuations Scale [12]. The Hallucinations Parkinson's disease-associated 
psychotic symptoms questionnaire was used to evaluate the presence of hallucinations [13]. RBD was evaluated using a questionnaire based on the article by Gjerstad et al. [14], simplified into two questions for the patient and the caregiver, one concerning movements during sleep and the other concerning vivid dreams and nightmares.

Patients with AD were selected according to Albert's criteria [15] and Dubois' criteria [16] for patients with pro-AD and McKhann's criteria [17] and Dubois' criteria [16] for demented AD patients.

Patients were considered to have DLB and AD when they meet both the Dubois' criteria and the McKeith's criteria concurrently. For example, a patient with memory storage disorders, a CSF in favor of AD, and two of the four clinical criteria for DLB was considered to have both DLB and AD.

Table 1 summarizes the main clinical information of the patients at the time of lumbar puncture. A total of 166 patients were included in this study: 21 control subjects (CS group), 51 patients with DLB at the prodromal stage (pro-DLB group), 16 patients with DLB at the demented stage (DLB-d group), $33 \mathrm{AD}$ patients at the prodromal stage (pro-AD group), $32 \mathrm{AD}$ patients at the demented stage (AD-d group), and 13 patients with both the criteria of $\mathrm{AD}$ and criteria of probable DLB [3], divided into two groups (pro-AD/DLB group $[n=2]$ and AD/DLB-d group $[n=11])$; data of the latter two groups were analyzed separately from the data of patients with pure $A D$ or pure DLB (see flowchart in Fig. 1). The CS group consisted of patients originally included in the study with cognitive disorders as found in AD and DLB, who, after follow-up in the study, were found to have neither AD nor DLB. The CS group had various diagnoses, defined according to international criteria (for details, see Table 1).

\section{CSF samples and analysis}

CSF samples were obtained by lumbar puncture in the context of the AlphaLewyMA protocol in a diagnostic workup for suspected cognitive decline and underwent a standard protocol (i.e., they were collected in polypropylene tubes [Sarstedt, ref.: 62.610.201] to decrease adsorption of $A \beta$ into the test tubes). Each CSF sample was transported to the laboratory within $4 \mathrm{~h}$ after collection; the sample was homogenized on receipt at the laboratory and was then centrifuged at $1700 \mathrm{~g}$ for $10 \mathrm{~min}$ at room temperature. All samples were free of blood contamination (the samples were checked visually; if a stain in the sample was detected, the sample was not measured). Samples were then transferred in $0.5-\mathrm{mL}$ polypropylene tubes (Dutscher ref.: 033283) and stored at $-80^{\circ} \mathrm{C}$ until analysis. CSF $A \beta 42, A \beta 40, t-T a u$, and phospho-tau 181 were measured by sandwich enzyme-linked immunosorbent assay (ELISA) using commercially available kits (INNOTEST ${ }^{\circ}$;
Fujirebio Europe, Ghent, Belgium). All assays were performed according to the manufacturer's instructions, and the methodology did not change during the period in which the analyses were performed. Note that for $A \beta 1-$ 40 , we did not have the same number of patients as for the other biomarkers, either because the dosage was not done systematically or because there was insufficient CSF available to perform an additional $A \beta 40$ assay. For this parameter, 77 patients had a dosage of $A \beta 40$ and were distributed as follows: CS group: $n=10$, pro-DLB group: $n=$ 28, DLB-d group: $n=7$, pro-AD group: $n=17$, AD-d group: $n=9$, pro-AD/DLB group: $n=1$, and AD/DLB-d group: $n=5$.

These CSF assays were run as routine clinical neurochemical analyses by technicians trained in CSF analysis at the biochemistry laboratory of University Hospital of Strasbourg. Furthermore, the laboratory participates in the quality control (QC) worldwide program organized by the Alzheimer's Association QC program for CSF biomarkers. Of note, our results are acceptable in comparison with the other laboratories, thereby further ensuring the quality of the results. Moreover, two internal QC samples per parameter were included in ELISA tests to control for inter-assay variation. Inter-assay coefficients of variations were $2.5-8.7 \%$ for $\mathrm{A} \beta 42,4.4-8.3 \%$ for $\mathrm{t}-\mathrm{Tau}, 4.9-16.4 \%$ for phospho-Tau 181 , and $1.5-9.0 \%$ for $A \beta 40$. The intra-assay variability observed in replicates was less than $10 \%$ in all four biomarkers.

The cut-offs used were, therefore, for A $\beta 42,500 \mathrm{ng} / \mathrm{L}$ (reduced levels were considered pathological); for $\mathrm{t}$-Tau (depending on age), $300 \mathrm{ng} / \mathrm{L}$ ( $<50$ years old), $450 \mathrm{ng} / \mathrm{L}$ (50-70 years old), and $500 \mathrm{ng} / \mathrm{L}$ ( $>70$ years old); for phospho- $\mathrm{Tau}_{181}, 60 \mathrm{ng} / \mathrm{L}$; for t-Tau and phospho-Tau ${ }_{181}$, increased levels were considered pathological. For the ratio $\mathrm{A} \beta 42 / \mathrm{A} \beta 40$, the cut-off used was 0.05 ; reduced levels were considered pathological.

CSF levels of total $\alpha$-syn were assessed using a commercial ELISA for $\alpha$-syn (hSYN total ELISA; AJ Roboscreen $\mathrm{GmbH}$, Leipzig, Germany) designed and validated for quantification of total $\alpha$-syn in human CSF [19]. The assay uses a monoclonal capture antibody recognizing amino acids 119 to 126 and a detection antibody to the Cterminus of $\alpha$-syn. Linearity of the assay is described between 50 and $600 \mathrm{pg} / \mathrm{mL}$. Intra-assay variability of $4.5 \%$ was calculated from duplicate analyses and expressed as median of the range to average of the duplicates. Interassay imprecision was determined using two qualitycontrol CSF pool samples, low control $10.5 \%$ and high control $3.7 \%$.

\section{Statistical analysis}

Statistical analyses were carried out using Graph-Pad PRISM, V.8 (GraphPad, San Diego, CA, USA). Normally distributed data were analyzed using one-way analysis of 


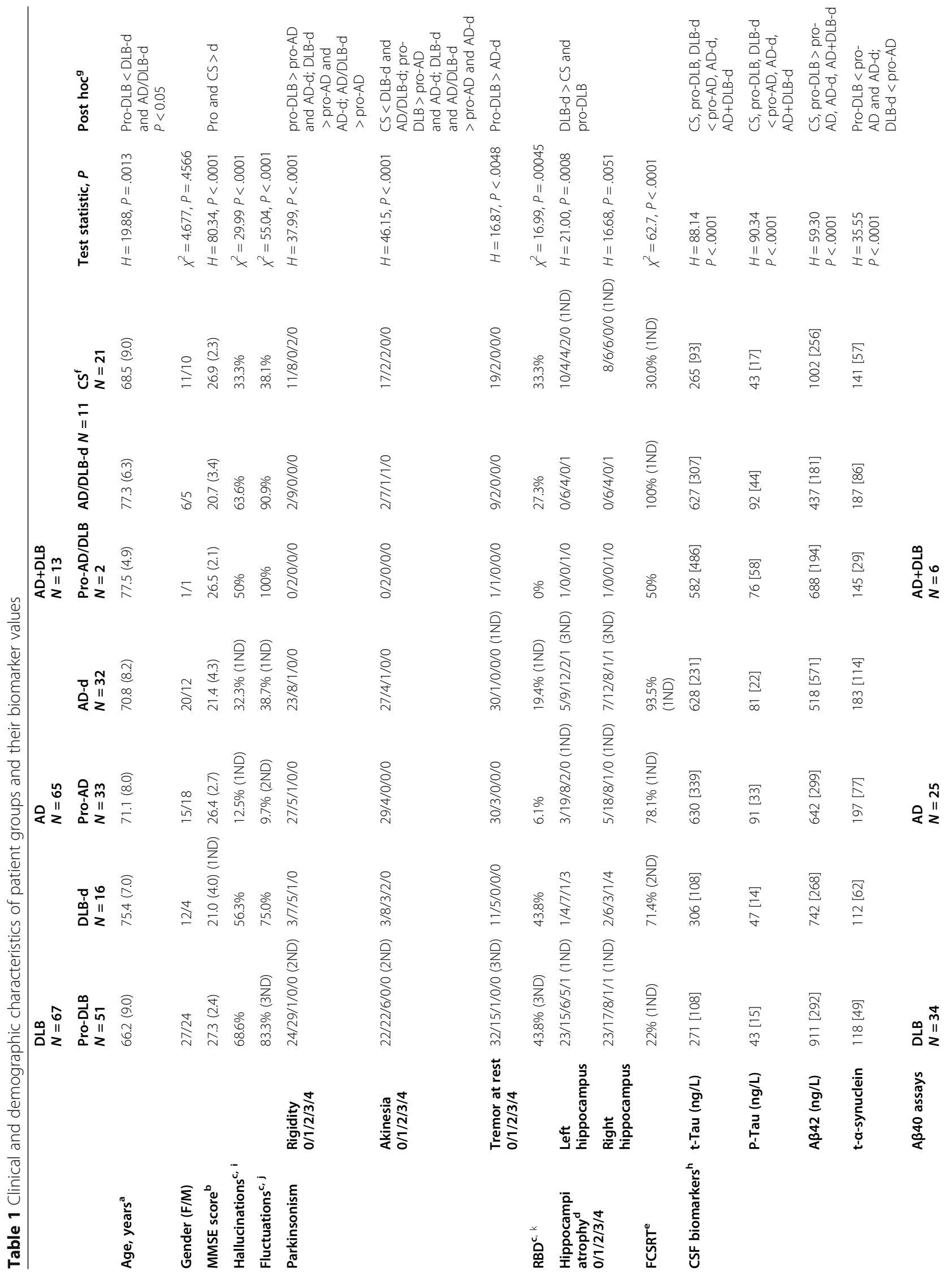




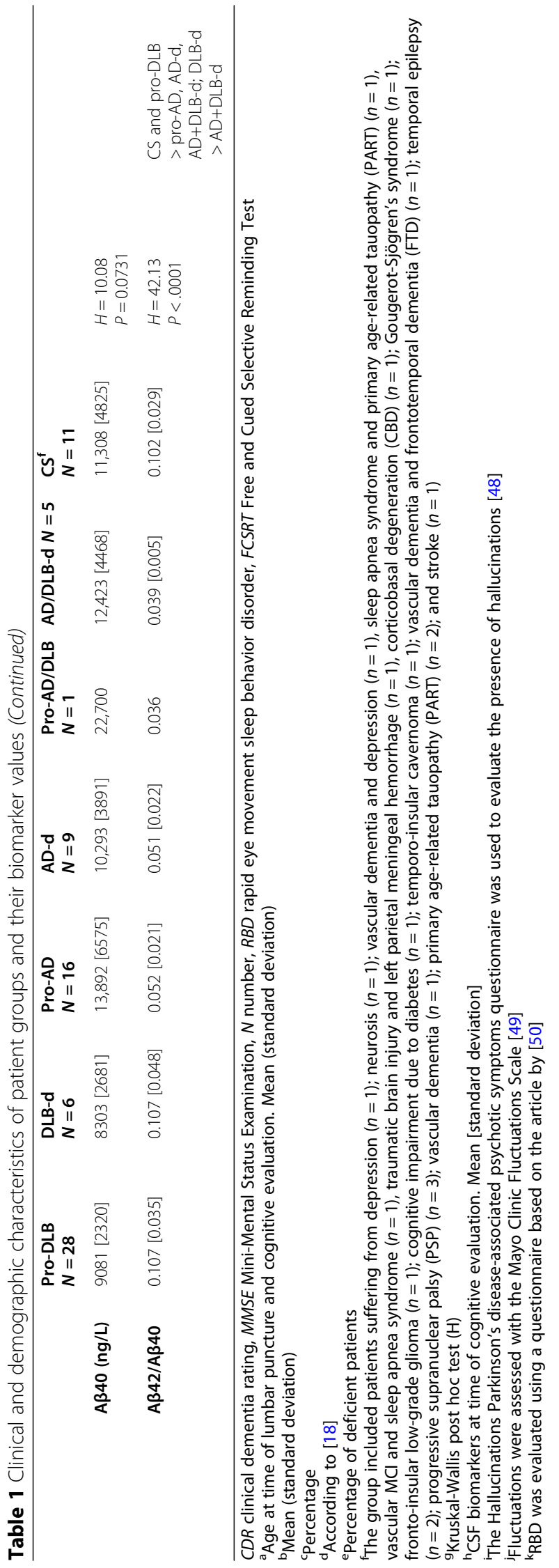




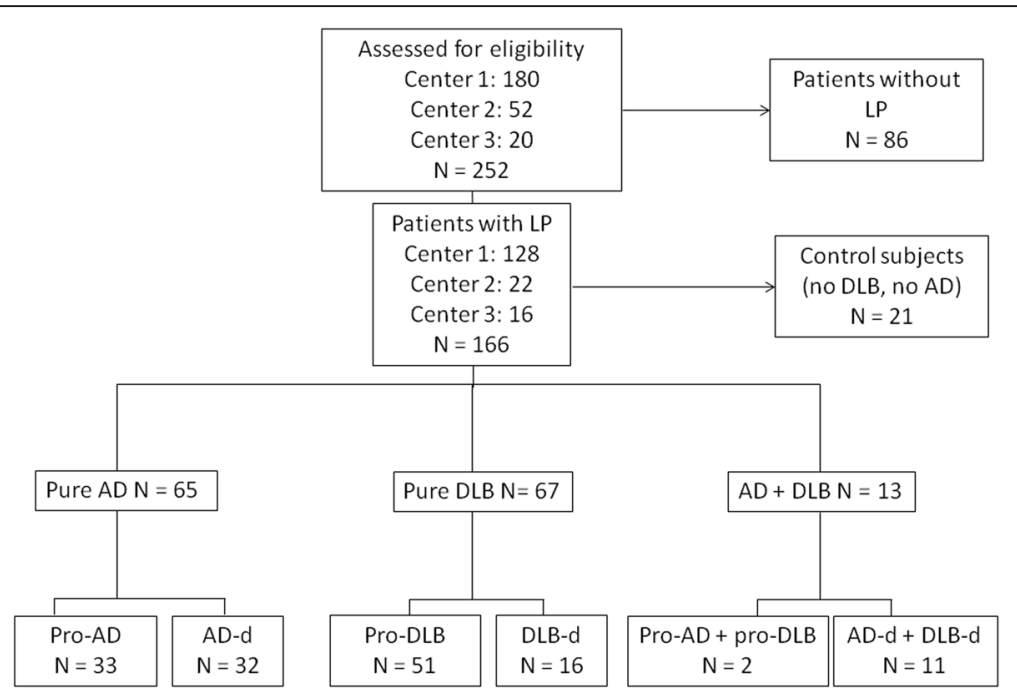

Fig. 1 Flowchart of patient selection from AlphaLewyMA study. Center 1: CHU de Hautepierre; center 2: Hôpital de la Robertsau; center 3: Hôpitaux Civils de Colmar; AD, Alzheimer's disease; DLB, dementia with Lewy bodies; Pro-AD, prodromal AD; Pro-DLB, prodromal DLB; AD-d, AD demented; DLB-d, DLB demented

variance with Tukey's post hoc analyses to determine between-group differences. In the case of non-Gaussiandistributed parameters, we used the Kruskal-Wallis test with Dunn's multiple comparison test. In the case of contingency analyses, a $\chi^{2}$ test was used. Receiver-operating characteristic (ROC) curve analysis was employed to evaluate the diagnostic value of CSF parameters. ROC curve comparisons were performed using MedCalc, V.12.7.0 (MedCalc Software, Ostend, Belgium).

\section{Results}

The study population's demographic characteristics and mean CSF biomarker values $(A \beta 42, A \beta 40, t-T a u$, phospho- $\mathrm{Tau}_{181}$, and $\alpha$-syn) are presented in Table 1 . It should be noted that for the comparison of the different parameters studied, the pro-AD/DLB group was excluded from the analyses due to the small number of patients. In summary, for $\mathrm{t}-\mathrm{Tau}$, the pro- $\mathrm{AD}, \mathrm{AD}-\mathrm{d}$, and AD/DLB-d groups had higher values compared to the CS, pro-DLB, and DLB-d groups (see Table 1). For P$\mathrm{Tau}$, the profile was very similar to that of $\mathrm{t}$-Tau. For A 442 , there was no significant difference between the CS group and the pro-DLB group but these two groups were significantly different from the pro-AD, AD-d, and AD/DLB-d groups, which all had lower values. However, the DLB-d group was not significantly different from the $\mathrm{CS}$, pro-DLB, pro-AD, $\mathrm{AD}-\mathrm{d}$, and $\mathrm{AD} / \mathrm{DLB}-\mathrm{d}$ groups. For $A \beta 40$, there were no differences between the groups. The ratio $A \beta 42 / A \beta 40$ was not significantly different between the CS, pro-DLB, and DLB-d groups; mean values for the pro-DLB group were significantly higher when compared to each of the AD groups (pro-AD, AD-d, and $A D / D L B-d$ ) and those of the CS group were significantly higher compared to the pro-AD and AD/DLB-d groups, whereas those of the DLB-d group were not significantly different from each of the other groups (Table 1).

\section{a-syn biomarker profile}

The results of the $\alpha$-syn assay are presented in Fig. 2a. No differences were observed between the CS and any of the other groups. $\alpha$-syn values were similar between the pro-DLB and DLB-d groups and between the pro$A D, A D-d$, and $A D / D L B-d$ groups. Interestingly, there was a significant difference between the DLB and $A D$ groups (pro-AD $>$ pro-DLB and DLB-d, $P<0.001$; AD$\mathrm{d}>$ pro-DLB, $P<0.05$ ).

Thus, we observed that the changes in $\alpha$-syn levels according to pathologies (AD or DLB) appeared from the prodromal stages. For this reason, to discriminate between $\mathrm{AD}$ and $\mathrm{DLB}$, the analysis of the diagnostic efficacy of $\alpha$-syn by the ROC curve, we have pooled the prodromal stages with the demented stages (Fig. 2b, c). The discrimination power of $\alpha$-syn between the 2 diseases remains moderate $(\mathrm{AUC}=0.78, \mathrm{Se}=72.3$ and $\mathrm{Sp}=$ 76.1 for a $139 \mathrm{ng} / \mathrm{L}$ criterion) (Fig. 2b, c and Table 2).

\section{Biomarker combinations}

Even if the discrimination power of total $\alpha$-syn seems moderate, it is interesting to determine if, combined with Alzheimer biomarkers, it improves this discrimination power between these two pathologies. As we have previously shown $[9,10]$, the $\mathrm{t}$-Tau, phospho-Tau, and $A \beta 42$ combination was very effective in discriminating between these two diseases (AUC $=0.95$ for DLB-(pro+ d) vs $\mathrm{AD}$-(pro+d); Table 2), but unfortunately, the addition of $\alpha$-syn did not improve this differential 

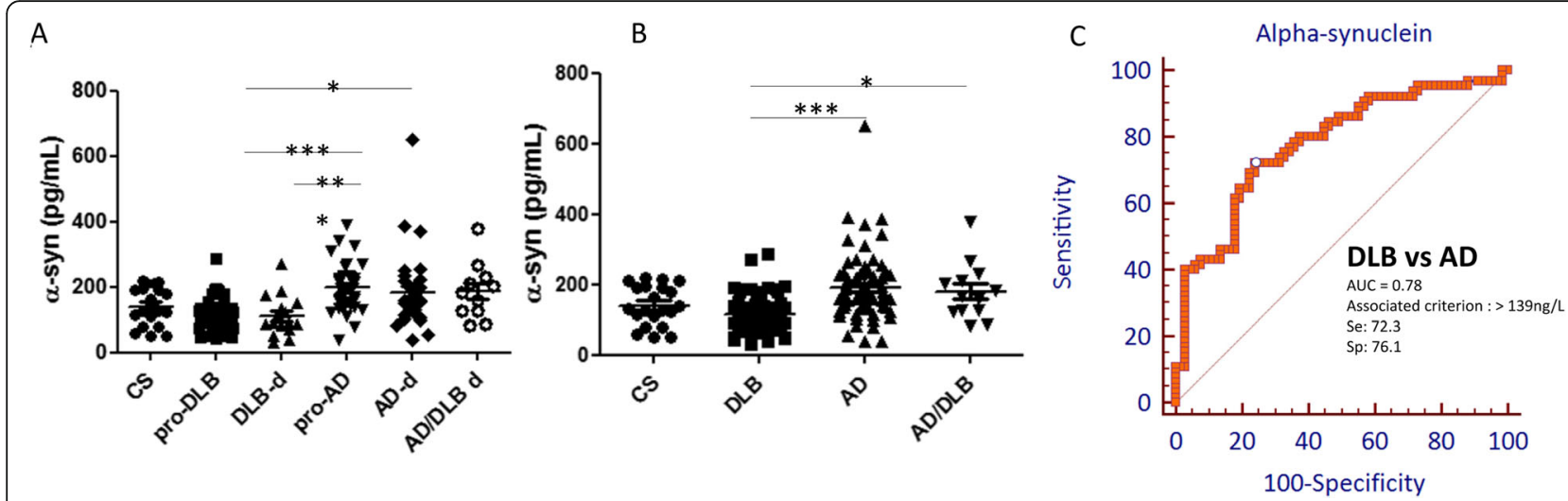

Fig. 2 Total alpha-synuclein assay discriminates between AD and DLB (a) and $\mathbf{b}$ scatterplots of CSF alpha-synuclein. a CSF concentrations of total alpha-synuclein in each patient group (the number of patients per group was as follows: CS $n=21$, pro-DLB $n=51$, DLB-d $n=16$, pro-AD $n=33$, AD-d $n=32$, and AD/DLB-d $n=11$ ) and $\mathbf{b}$ CSF concentration of alpha-synuclein in CS, DLB (pro-DLB + DLB-d), AD (pro-AD + AD-d), and AD/DLB (mixed pathologies: pro-AD/DLB + AD/DLB-d). ${ }^{* *} P<0.001 ;{ }^{*} P<0.05$. $P$ values were calculated using the Kruskal-Wallis test with Dunn's multiple comparison test. c Alpha-synuclein ROC curve between DLB and AD groups. Prodromal and demented patients were pooled in each group. Number of patients per group: DLB $n=67, A D n=65$. Se, sensitivity; Sp, specificity

diagnosis $(\mathrm{AUC}=0.95$ for DLB-(pro+d) vs AD-(pro+d); Table 2); the same applies if $A \beta 42$ is replaced by the ratio $\mathrm{A} \beta 42 / \mathrm{A} \beta 40$ (t-Tau_phospho-Tau_A $\beta 42 / \mathrm{A} \beta 40$ AUC = $0.95 ; \mathrm{t}-\mathrm{Tau} \_$phospho-Tau_A $\beta 42 / \mathrm{A} \beta 40 \_\mathrm{t}-\alpha$-syn $\mathrm{AUC}=$ 0.95 for DLB-(pro+d) vs AD-(pro+d); Table 2).

\section{Discussion}

In summary, the power of $\alpha$-syn to discriminate between $\mathrm{AD}$ and DLB can be considered moderate (Table 2), as previously reported $[20,21]$. However, our study shows that the differences observed between AD and DLB appear from the prodromal stage.

Our study has a limitation in that we do not know the exact concentration of hemoglobin in our samples. Indeed, it has been shown that hemoglobin plays a role in $\alpha$-syn levels in the CSF [22-25]. These studies have shown that beyond $200-500 \mathrm{ng} / \mathrm{mL}$ (depending on the study) hemoglobin leads to an artificial increase by interfering with the $\alpha$-syn assay. However, our samples were visually inspected upon arrival at the laboratory and any samples with pink coloration due to the presence of hemoglobin were rejected. This control is reported to eliminate hemorrhagic samples with more than 500 red cells per $\mu \mathrm{L}$ [26]. Furthermore, on arrival at the laboratory, samples were centrifuged at $1700 \mathrm{~g}$ for $10 \mathrm{~min}$ to eliminate as many blood cells as possible that could have contaminated the CSF, thus limiting hemoglobin levels in our samples.

\section{Early modification of $a$-syn levels}

Regarding the results of the total $\alpha$-syn assay, we found a significant difference between the DLB group and the AD group. Similar results have previously been highlighted in many publications [20, 21, 26-31], with $\alpha$-syn levels being higher in $\mathrm{AD}$ patients compared to DLB patients. These results have even been confirmed in an autopsy series of patients [32].

The originality of our results is to show that, at the prodromal stage, AD patients had significantly higher $\alpha$ syn levels than DLB patients. So far, only one recent publication has looked at the prodromal stage and has shown results similar to ours [33]; however, in that study, there were no patients at the demented stage. Thus, we have highlighted more precisely the absence of any change in $\alpha$-syn levels between the prodromal and dementia stages whatever the pathology (AD or DLB). Thus, total $\alpha$-syn levels are modified from the prodromal stages (Fig. 2a), suggesting that changes in $\alpha$-syn levels are implemented early.

\section{Ability of a-syn to discriminate between neurological controls and DLB and AD patients}

$\alpha$-syn levels of our control subjects were not significantly different from the AD and DLB groups, most likely because of the different neurological pathologies in this group, which made it heterogeneous. In the same way in the literature, it is usually the case that DLB patients were not significantly different from controls $[20,21,27$, $29,31,34-38$ ], but a number of publications showed significantly lower levels of $\alpha$-syn in DLB patients compared to control patients [30, 32, 39, 40]. Garcia-Ayllon et al. even showed that this decrease could take place from the DLB prodromal stage [33].

Interestingly, even if some studies, like ours, showed CSF $\alpha$-syn levels that were numerically higher, but not significantly so, in AD patients than in CS patients [24], most studies comparing CS patients and AD patients showed that total $\alpha$-syn levels were significantly higher 

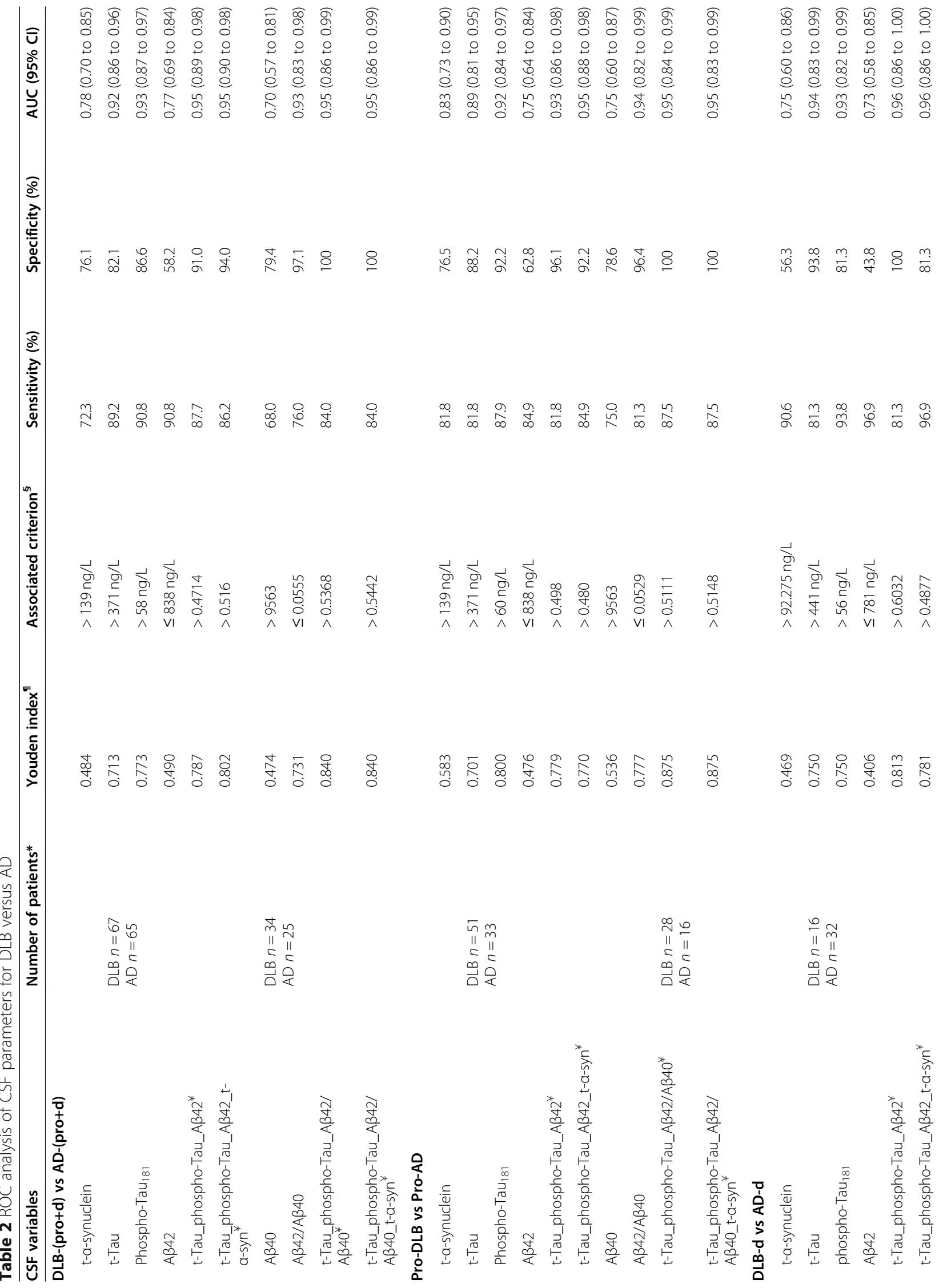


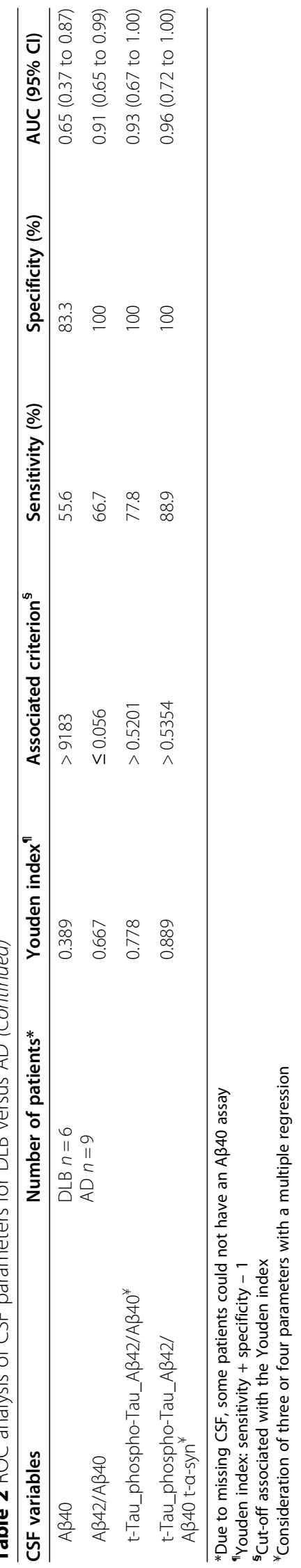


in $\mathrm{AD}$ patients [22, 24, 27, 30, 41], suggesting an $\alpha$-syn increase in $\mathrm{AD}$ patients. On the other hand, by observing the group of patients with AD+DLB comorbidity, it can be seen that the mean $\alpha$-syn values were at the same level as those of the pure AD groups. This result reinforces the idea that the change in $\alpha$-syn levels in the CSF is related to an $\alpha$-syn increase in AD rather than an $\alpha$-syn decrease in DLB. There are several possible explanations for this increase in $\mathrm{AD}$ patients. First, $\alpha$-syn could be released from damaged neurons [42, 43], as has been hypothesized for the increased levels of CSF tau in AD. Second, an increase in $\alpha$-syn production was confirmed by Larson et al., who highlighted a 1.67 -fold increase in $\alpha$-syn mRNA levels in the inferior temporal gyrus of $\mathrm{AD}$ patients, when compared to age-matched controls, leading to an increase in $\alpha$-syn monomers even though these $\mathrm{AD}$ patients did not have detectable Lewy bodies [44]. Thus, the increase in $\alpha$-syn production in the brains of $\mathrm{AD}$ patients is believed to be responsible for its increase in CSF. In addition, it has been shown that high levels of $\alpha$-syn may cause cognitive deficits by reducing the release of neurotransmitters by inhibiting the recycling of synaptic vesicles [45]. Thus, it is likely that these increases in soluble $\alpha$-syn (even in the monomeric form) in the brains of $\mathrm{AD}$ patients are the source of an important correlate of decreased cognitive function in $\mathrm{AD}$.

As DLB patients also have neuronal damage, it may seem surprising that there is no $\alpha$-syn increase in DLB patients. There are two possible explanations for this. First, the aggregating processes of $\alpha$-syn present in DLB patients are responsible for the decrease in $\alpha$-syn levels in the CSF, as observed for A $\beta 42$ in AD. The second explanation is that for the same level of cognitive impairment, DLB patients have less neurodegeneration than AD patients $[46,47]$, which may explain the lower value in DLB patients.

\section{The different proteinopathies have synergistic adverse effects}

Thus, while AD patients have amyloid plaques and DLB patients have Lewy bodies, CSF of AD patients presents an $\alpha$-syn level increase and CSF of DLB patients an A 342 decrease. These results indicate that these pathologies seem to be related in one way or another, which would explain the high frequency of comorbidities, or at least histological hallmarks commonly found between these 2 pathologies. More than $80 \%$ of DLB patients showed moderate or abundant cortical amyloid plaques [48], and $\alpha$-synuclein pathology is also found in up to $50 \%$ of patients with AD (for a review, see [49]), suggesting a close link between amyloidopathy and synucleinopathy. In addition, other publications indicate that Tau protein may also have a negative synergy with amyloidopathy and synucleinopathy [50, 51], reinforcing the close link between these different neurodegenerative diseases.

\section{Ability of the combination of a-syn with standard AD- related biomarkers to discriminate DLB from AD}

ROC curves (Table 2) show that even combining $\alpha$-syn results with Alzheimer biomarkers does not improve the discrimination power compared to the combination of Alzheimer biomarkers alone (t-Tau_phospho-Tau_A $\beta 42$ or $\mathrm{A} \beta 42 / \mathrm{A} \beta 40$, AUC $=0.95$, Alzheimer biomarkers $+\alpha$ syn $A U C=0.95)$. However, this result needs to be put into perspective given that the CSF's Alzheimer biomarkers are taken into account in the diagnosis, leading to a bias due to an overestimation of the discrimination effectiveness of these Alzheimer biomarkers. Despite taking into account the CSF result, some patients, particularly those clinically considered as Alzheimer's, present an atypical CSF profile. However, we are quite confident in the diagnosis; in fact, some patients have started to be included in the study from 2013, and consequently, we have a relatively long follow-up of these patients, which has allowed us to reclassify some of them.

\section{Conclusions}

To conclude, the total $\alpha$-syn assay can participate to discriminate between DLB and AD patients, whatever the stage, but with insufficient specificity and sensitivity. Thus, there is currently a clear lack of new biomarkers specific to DLB for its differential diagnosis. However, other biomarkers are under study. While some are directly related to $\alpha$-syn, such as the $\alpha$-syn oligomers, fibrils, or phosphorylation on S129 of $\alpha$-syn, there are other post-translational modifications or even biomarkers which are unrelated to the direct aggregation processes of $\alpha$-syn, such as YKL-40, neurogranin, and VILIP-1 (for review, see [8]); yet these biomarkers suffer from a lack of hindsight to determine if they are actually relevant in the biological diagnosis of DLB. Further studies are therefore needed to confirm these results.

\section{Abbreviations \\ a-syn: Alpha-synuclein; AD: Alzheimer's disease; AD-d: AD demented; \\ CS: Control subjects; CSF: Cerebrospinal fluid; DLB: Dementia with Lewy bodies; DLB-d: DLB demented; FCSRT: Free and Cued Selective Reminding Test; MCl: Mild cognitive impairment; MMSE: Mini-Mental State Examination; Phospho-Tau 181 : Tau phosphorylated on residue 181; Pro-AD: Prodromal AD; Pro-DLB: Prodromal DLB; RBD: Rapid eye movement sleep behavior disorder; TMT: Trail Making Test (A or B); t-Tau: Total Tau}

\section{Acknowledgements}

The authors would especially like to thank Analytik Jena Roboscreen for graciously donating the alpha-synuclein ELISA kits.

Authors' contributions

$\mathrm{OB}, \mathrm{TL}, \mathrm{APL}$, and FB: study concept and design, analysis of the results, and drafting the manuscript. OB and TL: statistical analyses. CSA: biological 
measurements. IL: supplier of alpha-synuclein ELISA kits for the company AJ Roboscreen. APL: organization and analyses of the alpha-synuclein kit validation. $\mathrm{OB}, \mathrm{TL}, \mathrm{APL}$, and $\mathrm{IL}$ : analyses of biological measurements and contribution to data interpretation and revision of the manuscript for important intellectual content. $A B, L R, B J$, and FB: study protocol design. PLS: implementation and management of MRI acquisitions. NP, PA, CD, CMH, BC, and FB: clinical work, organization of lumbar punctures, diagnosis confirmation, and contribution to data interpretation and revision of the manuscript for important intellectual content. The author(s) read and approved the final manuscript.

\section{Funding}

PHRC Interégional

\section{Availability of data and materials}

All data generated or analyzed during this study are included in this published article.

\section{Ethics approval and consent to participate}

CPP Est IV, Eudract 2012-A00992-41 / HUS 5330.

\section{Consent for publication}

Not applicable

\section{Competing interests}

Ingolf Lachmann provided the ELISA alpha-synuclein kits and reports that he is an employee of AJ Roboscreen $\mathrm{GmbH}^{\oplus}$, Leipzig, Germany.

\section{Author details}

'Laboratory of Biochemistry and Molecular Biology, University Hospital of Strasbourg, 67000 Strasbourg, France. ${ }^{2}$ Laboratoire de Neurosciences Cognitives et Adaptatives (LNCA), University of Strasbourg, 67000 Strasbourg, France. ${ }^{3}$ CNRS UMR7364, 67000 Strasbourg, France. ${ }^{4}$ CM2R (Research and Resources Memory (entre), Geriatric Day Hospital and Neuropsychology Unit, Geriatrics Department, University Hospitals of Strasbourg, Strasbourg, France. ${ }^{5}$ University of Strasbourg and CNRS, ICube Laboratory UMR 7357 and FMTS (Fédération de Médecine Translationnelle de Strasbourg), Team IMIS, Strasbourg, France. ${ }^{6}$ Neurochemistry Laboratory, Biochemistry Department, Centre de Biologie et Pathologie Est, Hospices Civils de Lyon, 59 bd Pinel, 69677 Bron, France. 'BIORAN Team, Lyon Neuroscience Research Center, CNRS UMR 5292 - INSERM U1028, Université de Lyon - Université Claude Bernard, 95 bd Pinel, 69675 Bron, France. ${ }^{8}$ Center for Memory Resources and Research, Hospices Civils de Lyon, Charpennes Hospital, Lyon 1 University, 69100 Villeurbanne, France. ${ }^{9}$ AJ Roboscreen GmbH, Hohmannstraße 7, 04129 Leipzig, Germany. ${ }^{10}$ Geriatrics Department, General Hospital Centre, CM2R, Geriatric Day Hospital, Colmar, France.

Received: 20 March 2020 Accepted: 10 September 2020 Published online: 29 September 2020

\section{References}

1. McKeith IG, Galasko D, Kosaka K, Perry EK, Dickson DW, Hansen LA, et al. Consensus guidelines for the clinical and pathologic diagnosis of dementia with Lewy bodies (DLB): report of the consortium on DLB international workshop. Neurology. 1996;47(5):1113-24.

2. McKeith IG, Ballard CG, Perry RH, Ince PG, O'Brien JT, Neill D, et al. Prospective validation of consensus criteria for the diagnosis of dementia with Lewy bodies. Neurology. 2000:54(5):1050-8.

3. McKeith IG, Dickson DW, Lowe J, Emre M, O'Brien JT, Feldman H, et al. Diagnosis and management of dementia with Lewy bodies: third report of the DLB Consortium. Neurology. 2005;65(12):1863-72.

4. McKeith IG, Boeve BF, Dickson DW, Halliday G, Taylor JP, Weintraub D, et al. Diagnosis and management of dementia with Lewy bodies: fourth consensus report of the DLB Consortium. Neurology. 2017;89(1):88-100.

5. Nelson PT, Jicha GA, Kryscio RJ, Abner EL, Schmitt FA, Cooper G, et al. Low sensitivity in clinical diagnoses of dementia with Lewy bodies. J Neurol. 2010;257(3):359-66.

6. McKeith IG, Ferman TJ, Thomas AJ, Blanc F, Boeve BF, Fujishiro H, et al Research criteria for the diagnosis of prodromal dementia with Lewy bodies. Neurology. 2020;94(17):743-55.
7. Jellinger KA, Attems J. Prevalence and pathology of dementia with Lewy bodies in the oldest old: a comparison with other dementing disorders. Dement Geriatr Cogn Disord. 2011;31(4):309-16.

8. Bousiges O, Blanc F. Diagnostic value of cerebro-spinal fluid biomarkers in dementia with lewy bodies. Clin Chim Acta. 2019;490:222-8.

9. Bousiges O, Cretin B, Lavaux T, Philippi N, Jung B, Hezard S, et al. Diagnostic value of cerebrospinal fluid biomarkers (phospho-Tau181, total-tau, Abeta42, and Abeta40) in prodromal stage of Alzheimer's disease and dementia with Lewy bodies. J Alzheimers Dis. 2016;51(4):1069-83.

10. Bousiges O, Bombois S, Schraen S, Wallon D, Quillard MM, Gabelle A, et al. Cerebrospinal fluid Alzheimer biomarkers can be useful for discriminating dementia with Lewy bodies from Alzheimer's disease at the prodromal stage. J Neurol Neurosurg Psychiatry. 2018;89(5):467-75.

11. Kemp J, Philippi N, Phillipps C, Demuynck C, Albasser T, Martin-Hunyadi C, et al. Cognitive profile in prodromal dementia with Lewy bodies. Alzheimers Res Ther. 2017:9(1):19.

12. Ferman TJ, Boeve BF, Smith GE, Lin SC, Silber MH, Pedraza O, et al. Inclusion of RBD improves the diagnostic classification of dementia with Lewy bodies. Neurology. 2011;77(9):875-82.

13. Fenelon G, Soulas T, Zenasni F, Cleret de Langavant $L$. The changing face of Parkinson's disease-associated psychosis: a cross-sectional study based on the new NINDS-NIMH criteria. Mov Disord 2010;25(6):763-766.

14. Gjerstad MD, Boeve B, Wentzel-Larsen T, Aarsland D, Larsen JP. Occurrence and clinical correlates of REM sleep behaviour disorder in patients with Parkinson's disease over time. J Neurol Neurosurg Psychiatry. 2008;79(4): 387-91.

15. Albert MS, DeKosky ST, Dickson D, Dubois B, Feldman HH, Fox NC, et al. The diagnosis of mild cognitive impairment due to Alzheimer's disease: recommendations from the National Institute on Aging-Alzheimer's Association workgroups on diagnostic guidelines for Alzheimer's disease. Alzheimers Dement. 2011;7(3):270-9.

16. Dubois B, Feldman HH, Jacova C, Dekosky ST, Barberger-Gateau P, Cummings J, et al. Research criteria for the diagnosis of Alzheimer's disease: revising the NINCDS-ADRDA criteria. Lancet Neurol. 2007;6(8):734-46.

17. McKhann GM, Knopman DS, Chertkow H, Hyman BT, Jack CR Jr, Kawas CH, et al. The diagnosis of dementia due to Alzheimer's disease: recommendations from the National Institute on Aging-Alzheimer's Association workgroups on diagnostic guidelines for Alzheimer's disease. Alzheimers Dement. 2011;7(3):263-9.

18. Scheltens P, Leys D, Barkhof F, et al. Atrophy of medial temporal lobes on MRI in "probable" Alzheimer's disease and normal ageing: diagnostic value and neuropsychological correlates. J Neurol Neurosurg Psychiatry. 1992; 55(10):967-72. https://doi.org/10.1136/jnnp.55.10.967.

19. Lerche S, Machetanz G, Wurster I, Roeben B, Zimmermann M, Pilotto A, et al. Dementia with Lewy bodies: GBA1 mutations are associated with cerebrospinal fluid alpha-synuclein profile. Mov Disord. 2019;34(7):1069-73.

20. Hall S, Ohrfelt A, Constantinescu R, Andreasson U, Surova Y, Bostrom F, et al. Accuracy of a panel of 5 cerebrospinal fluid biomarkers in the differential diagnosis of patients with dementia and/or parkinsonian disorders. Arch Neurol. 2012;69(11):1445-52.

21. Wennstrom M, Surova $Y$, Hall S, Nilsson C, Minthon L, Bostrom F, et al. Low CSF levels of both alpha-synuclein and the alpha-synuclein cleaving enzyme neurosin in patients with synucleinopathy. PLoS One. 2013;8(1):e53250.

22. Korff A, Liu C, Ginghina C, Shi M, Zhang J. Alzheimer's disease neuroimaging I. alpha-synuclein in cerebrospinal fluid of Alzheimer's disease and mild cognitive impairment. J Alzheimers Dis. 2013;36(4):679-88.

23. Hong Z, Shi M, Chung KA, Quinn JF, Peskind ER, Galasko D, et al. DJ-1 and alpha-synuclein in human cerebrospinal fluid as biomarkers of Parkinson's disease. Brain. 2010;133(Pt 3):713-26.

24. Shi M, Tang L, Toledo JB, Ginghina C, Wang H, Aro P, et al. Cerebrospinal fluid alpha-synuclein contributes to the differential diagnosis of Alzheimer's disease. Alzheimers Dement. 2018;14(8):1052-62.

25. Wang H, Stewart T, Toledo JB, Ginghina C, Tang L, Atik A, et al. A longitudinal study of total and phosphorylated alpha-synuclein with other biomarkers in cerebrospinal fluid of Alzheimer's disease and mild cognitive impairment. J Alzheimers Dis. 2018;61(4):1541-53.

26. Mollenhauer B, Cullen V, Kahn I, Krastins B, Outeiro TF, Pepivani I, et al. Direct quantification of CSF alpha-synuclein by ELISA and first cross-sectional study in patients with neurodegeneration. Exp Neurol. 2008;213(2):315-25.

27. Chiasserini D, Biscetti L, Eusebi P, Salvadori N, Frattini G, Simoni S, et al. Differential role of CSF fatty acid binding protein 3, alpha-synuclein, and 
Alzheimer's disease core biomarkers in Lewy body disorders and Alzheimer's dementia. Alzheimers Res Ther. 2017;9(1):52.

28. Kasuga K, Tokutake T, Ishikawa A, Uchiyama T, Tokuda T, Onodera O, et al. Differential levels of alpha-synuclein, beta-amyloid42 and tau in CSF between patients with dementia with Lewy bodies and Alzheimer's disease. J Neurol Neurosurg Psychiatry. 2010;81(6):608-10.

29. Luo X, Hou L, Shi H, Zhong X, Zhang Y, Zheng D, et al. CSF levels of the neuronal injury biomarker visinin-like protein-1 in Alzheimer's disease and dementia with Lewy bodies. J Neurochem. 2013;127(5):681-90.

30. Tateno F, Sakakibara R, Kawai T, Kishi M, Murano T. Alpha-synuclein in the cerebrospinal fluid differentiates synucleinopathies (Parkinson disease, dementia with Lewy bodies, multiple system atrophy) from Alzheimer disease. Alzheimer Dis Assoc Disord. 2012;26(3):213-6.

31. Hansson O, Hall S, Ohrfelt A, Zetterberg H, Blennow K, Minthon L, et al. Levels of cerebrospinal fluid alpha-synuclein oligomers are increased in Parkinson's disease with dementia and dementia with Lewy bodies compared to Alzheimer's disease. Alzheimers Res Ther. 2014;6(3):25.

32. Mollenhauer B, Locascio JJ, Schulz-Schaeffer W, Sixel-Doring F, Trenkwalder C, Schlossmacher MG. Alpha-Synuclein and tau concentrations in cerebrospinal fluid of patients presenting with parkinsonism: a cohort study. Lancet Neurol. 2011:10(3):230-40.

33. Garcia-Ayllon MS, Monge-Argiles JA, Monge-Garcia V, Navarrete F, CortesGomez MA, Sanchez-Paya J, et al. Measurement of CSF alpha-synuclein improves early differential diagnosis of mild cognitive impairment due to Alzheimer's disease. J Neurochem. 2019:150(2):218-30.

34. Ohrfelt A, Grognet P, Andreasen N, Wallin A, Vanmechelen E, Blennow K, et al. Cerebrospinal fluid alpha-synuclein in neurodegenerative disorders-a marker of synapse loss? Neurosci Lett. 2009;450(3):332-5.

35. Kapaki E, Paraskevas GP, Emmanouilidou E, Vekrellis K. The diagnostic value of CSF alpha-synuclein in the differential diagnosis of dementia with Lewy bodies vs. normal subjects and patients with Alzheimer's disease. PLoS One. 2013;8(11):e81654.

36. Aerts MB, Esselink RA, Abdo WF, Bloem BR, Verbeek MM. CSF alphasynuclein does not differentiate between parkinsonian disorders. Neurobiol Aging. 2012;33(2):430 e1-3.

37. Reesink FE, Lemstra AW, van Dijk KD, Berendse HW, van de Berg WD, Klein $M$, et al. CSF alpha-synuclein does not discriminate dementia with Lewy bodies from Alzheimer's disease. J Alzheimers Dis. 2010;22(1):87-95.

38. Schmitz M, Villar-Pique A, Llorens F, Gmitterova K, Hermann P, Varges D, et al. Cerebrospinal fluid total and phosphorylated alpha-synuclein in patients with Creutzfeldt-Jakob disease and synucleinopathy. Mol Neurobiol. 2019:56(5):3476-83.

39. Parnetti L, Chiasserini D, Eusebi P, Giannandrea D, Bellomo G, De Carlo C, et al. Performance of abeta1-40, abeta1-42, total tau, and phosphorylated tau as predictors of dementia in a cohort of patients with mild cognitive impairment. J Alzheimers Dis. 2012;29(1):229-38.

40. Llorens F, Schmitz M, Varges D, Kruse N, Gotzmann N, Gmitterova K, et al. Cerebrospinal alpha-synuclein in alpha-synuclein aggregation disorders: tau/ alpha-synuclein ratio as potential biomarker for dementia with Lewy bodies. J Neurol. 2016;263(11):2271-7.

41. Slaets S, Le Bastard N, Martin JJ, Sleegers K, Van Broeckhoven C, De Deyn PP, et al. Cerebrospinal fluid Abeta1-40 improves differential dementia diagnosis in patients with intermediate P-tau181P levels. J Alzheimers Dis. 2013;36(4):759-67.

42. Bellani S, Sousa VL, Ronzitti G, Valtorta F, Meldolesi J, Chieregatti E. The regulation of synaptic function by alpha-synuclein. Commun Integr Biol. 2010;3(2):106-9.

43. Scott DA, Tabarean I, Tang Y, Cartier A, Masliah E, Roy S. A pathologic cascade leading to synaptic dysfunction in alpha-synuclein-induced neurodegeneration. J Neurosci. 2010;30(24):8083-95.

44. Larson ME, Sherman MA, Greimel S, Kuskowski M, Schneider JA, Bennett DA, et al. Soluble alpha-synuclein is a novel modulator of Alzheimer's disease pathophysiology. J Neurosci. 2012;32(30):10253-66.

45. Nemani VM, Lu W, Berge V, Nakamura K, Onoa B, Lee MK, et al. Increased expression of alpha-synuclein reduces neurotransmitter release by inhibiting synaptic vesicle reclustering after endocytosis. Neuron. 2010;65(1):66-79.

46. Harper L, Fumagalli GG, Barkhof F, Scheltens P, O'Brien JT, Bouwman F, et al. MRI visual rating scales in the diagnosis of dementia: evaluation in 184 post-mortem confirmed cases. Brain. 2016;139(Pt 4):1211-25.

47. Blanc F, Colloby SJ, Philippi N, de Petigny X, Jung B, Demuynck C, et al. Cortical thickness in dementia with Lewy bodies and Alzheimer's disease: a comparison of prodromal and dementia stages. PLoS One. 2015;10(6): e0127396.

48. Ballard C, Ziabreva I, Perry R, Larsen JP, O'Brien J, McKeith I, et al. Differences in neuropathologic characteristics across the Lewy body dementia spectrum. Neurology. 2006;67(11):1931-4.

49. Irwin DJ, Lee VM, Trojanowski JQ. Parkinson's disease dementia: convergence of alpha-synuclein, tau and amyloid-beta pathologies. Nat Rev Neurosci. 2013;14(9):626-36.

50. Giasson BI, Lee VM, Trojanowski JQ. Interactions of amyloidogenic proteins. NeuroMolecular Med. 2003;4(1-2):49-58.

51. Lee VM, Giasson Bl, Trojanowski JQ. More than just two peas in a pod: common amyloidogenic properties of tau and alpha-synuclein in neurodegenerative diseases. Trends Neurosci. 2004;27(3):129-34.

\section{Publisher's Note}

Springer Nature remains neutral with regard to jurisdictional claims in published maps and institutional affiliations.
Ready to submit your research? Choose BMC and benefit from:

- fast, convenient online submission

- thorough peer review by experienced researchers in your field

- rapid publication on acceptance

- support for research data, including large and complex data types

- gold Open Access which fosters wider collaboration and increased citations

- maximum visibility for your research: over $100 \mathrm{M}$ website views per year

At BMC, research is always in progress.

Learn more biomedcentral.com/submissions 